Research Article

\title{
Analysis Method for the Response of Structures Subjected to Non-stationary Seismic Excitations
}

\author{
Rui Kang ${ }^{1}$, Jin Zhang ${ }^{1 *}$, Liu-Xi Zhou ${ }^{1}$, Shi-Qiang Qin $^{2}$, Kai-Feng Zheng ${ }^{1}$ and De-Yi Zhang ${ }^{3}$ \\ ${ }^{1}$ School of Civil Engineering, Southwest Jiaotong University, Chengdu 610031, China \\ ${ }^{2}$ School of civil engineering and architecture, Wuhan University of Technology, Wuhan 430070, China \\ ${ }_{3}^{3}$ Department of Civil and Environmental Engineering, University of Waterloo, Waterloo, Canada
}

Received 10 May 2016; Accepted 29 June 2016

\begin{abstract}
A fast analysis approach was developed to improve the computational efficiency of seismic analysis for structures under non-stationary seismic excitations. A high-pier railway bridge was selected as a case study to evaluate the proposed fast approach. First, non-stationary excitation was translated into a series of deterministic transient analyses. Second, a modified high-precision integration method was introduced to reduce the number of transient analyses to two. Finally, a seismic response analysis of the high-pier railway bridge subjected to non-stationary ground motions was conducted with the secondary development platform of the general finite element software ANSYS. The fast analysis technique and ANSYS were combined. The results show the proposed fast analysis technique can model seismic non-stationary and spatial variability and can be easily extended to practical engineering applications.
\end{abstract}

Keywords: non-stationary excitation, high-precision integration, multi-dimension and multi-support, seismic spatial effect

\section{Introduction}

Many scholars have conducted detailed studies on calculation theory and its application to the dynamic responses of complex structures under non-stationary stochastic earthquake excitation. Numerous findings have been obtained, and these findings play an important role in promoting the uses of the stochastic vibration method of non-stationary excitation in practical engineering applications [1-4]. Given the characteristic randomness of earthquakes, the random vibration method (RVM) is widely utilized and accepted in the seismic analysis of long-span structures and has been adopted by several seismic design standards [5-7]. Lin [8] proposed the pseudo-excitation method (PEM), which is also known as the fast complete quadratic combination method, because RVM requires extensive computation for the seismic analysis of structures under tri-directional stochastic excitations. With the emergence of complex structures, further improvement of the computational efficiency of a complex structural dynamic response under non-stationary seismic excitation is necessary.

\section{State of the art}

Most previous scholars adopted advanced pseudo-excitation methods to deal with non-stationary seismic excitation [1-3].

\footnotetext{
* E-mail address: syhelen1989@163.com

ISSN: 1791-2377 @ 2016 Eastern Macedonia and Thrace Institute of Technology. All rights reserved.
}

Computational efficiency was improved by several orders of magnitude; however, given that finite element software lacked calculation modules for pseudo-excitation method, engineers had to design a program for the pre-treatment, calculation, and post-processing of the responses of structures subjected to non-stationary seismic excitation. To apply the computational theory of non-stationary seismic excitation in stochastic vibration methods to practical engineering even without programming in pre-treatment and post-processing, studies have been conducted on its applications in general finite element software, including whether stochastic vibration excitations from smooth to nonstationary can be realized in a general finite element software; this realization would extend ground motion excitation from 1D to 3D. Additionally, stationary ground motion would be fully considered in multi-support and multi-dimensional excitation analyses of large-span structures [9-11]. To fully consider the non-stationary nature of ground motion, the pseudo-excitation method with an absolute displacement direct approach has been utilized in literature [12]. Calculations under multi-support, multidimensional, and non-stationary conditions have been achieved in ANSYS and have provided the impetus for the consideration of the non-stationarity of ground motion. However, this method has low efficiency and requires a time history analysis of all discrete frequencies. Given this enormous workload, enhancing computational efficiency is a must. The structural dynamic response of a three-cross twelve-story frame structure under non-stationary excitation was calculated through explicit methods in previous studies [13-14]. The results indicated that the combination of timedomain explicit method and pseudo-excitation method significantly improves computational efficiency. However, these studies only considered lateral loads under uniform 
and non-uniform excitations, which are different from complex multi-dimensional multi-support seismic excitation. Therefore, the structural response under non-stationary seismic excitation requires further study.

A fast simulation method was developed in the current study. The method was implemented in ANSYS software. First, the structural dynamic response under non-stationary seismic excitation was deduced theoretically. Second, the theoretical solution of the response with the SDOF structure was compared with the results calculated by the proposed method. The results show that the proposed method is rational, efficient, and correct. Finally, a 3D numerical finite element model of a high-pier railway bridge located in western China was established in ANSYS to conduct a structural response analysis under non-stationary seismic excitation. The improved high-precision integration method can be applied to practical engineering.

\section{Methodology}

3.1 Pseudo-excitation method in seismic analysis of structures subjected to tri-directional seismic excitations The equations of motion for a discretized linear, n-degree-of freedom structural system subjected to $m$ directional support motions (three translational components) can be expressed in a partitioned matrix form in the global coordinate system as follows [15]:

$$
\left[\begin{array}{cc}
M_{s s} & M_{s b} \\
M_{b s} & M_{b b}
\end{array}\right]\left\{\begin{array}{l}
\ddot{X}_{s} \\
\ddot{X}_{b}
\end{array}\right\}+\left[\begin{array}{cc}
C_{s s} & C_{s b} \\
C_{b s} & C_{b b}
\end{array}\right]\left\{\begin{array}{c}
\dot{X}_{s} \\
\dot{X}_{b}
\end{array}\right\}+\left[\begin{array}{cc}
K_{s s} & K_{s b} \\
K_{b s} & K_{b b}
\end{array}\right]\left\{\begin{array}{c}
X_{s} \\
X_{b}
\end{array}\right\}=\left\{\begin{array}{c}
0 \\
P_{b}
\end{array}\right\}
$$

Where vector $\left\{X_{b}\right\}_{3 \times m}=\left\{X_{1}, X_{1}, \mathrm{~K}, X_{3 \times m}\right\}^{T}$ represents the enforced displacements of $\mathrm{m}$ supports, $\left\{X_{s}\right\}_{3 \times n}=\left\{X_{1}, X_{1}, \mathrm{~K}, X_{3 \times n}\right\}^{T}$ denotes the vector of absolute displacements of the slave degrees of freedom (DoFs), and $\left\{P_{b}\right\}_{3 \times m}=\left\{P_{1}, \mathrm{P}_{1}, \mathrm{~K}, \mathrm{P}_{3 \times m}\right\}^{T}$ is the vector of seismic forces at structural supports. $M, C$, and $\mathrm{K}$ are the mass, damping, and stiffness matrices, respectively. The subscripts $\mathrm{b}$ and $\mathrm{s}$ refer to the master and slave DoFs, respectively. $M_{b s}, C_{b s}$, and $K_{b s}$ denote the transpose of matrices $M_{s b}, C_{s b}$, and $K_{s b}$, respectively. The lumped mass model is assumed in this study, i.e., $M_{s b}$ is null.

Matrix manipulation in the second block row of Eq. (1) results in

$$
M_{b s} \cdot \ddot{X}_{s}+M_{b b} \cdot \ddot{X}_{b}+C_{b s} \cdot \dot{X}_{s}+C_{b} \cdot \dot{X}_{b}+K_{b s} \cdot X_{s}+K_{b} \cdot X_{b}=P_{b}
$$

where the vector of seismic forces, $P_{b}$, can be expressed in terms of the mass of supports $\boldsymbol{M}_{\mathrm{bb}}$ and ground acceleration $\ddot{\boldsymbol{u}}_{\mathrm{b}}$ as follows:

$$
P_{b}=M_{b b} \cdot \ddot{u}_{b}
$$

Eq. (2) can then be rewritten as

$$
M_{b s} \cdot \ddot{X}_{s}+M_{b b} \cdot \ddot{X}_{b}+C_{b s} \cdot \dot{X}_{s}+C_{b} \cdot \dot{X}_{b}+K_{b s} \cdot X_{s}+K_{b} \cdot X_{b}=M_{b b} \cdot \ddot{u}_{b}
$$

Multiplying $M_{b b}^{-1}$ on both sides of Eq. (4) results in

$$
\begin{aligned}
& M_{b b}^{-1}\left(M_{b s} \cdot \ddot{X}_{s}+M_{b b} \cdot \ddot{X}_{b}+C_{b s} \cdot \dot{X}_{s}++K_{b} \cdot X_{b}\right) \\
& =M_{b b}^{-1} \cdot M_{b b} \cdot \ddot{u}_{b}
\end{aligned}
$$

A very large value can be assigned to $\boldsymbol{M}_{\mathrm{bb}}$. Then, $M_{b b}^{-1} \rightarrow 0$, and $M_{b b}^{-1} \cdot M_{b s}, M_{b b}^{-1} \cdot C_{b s}, M_{b b}^{-1} \cdot C_{b b}, M_{b b}^{-1} \cdot K_{b s}, M_{b b}^{-1} \cdot K_{b b}$ are 0 . Accordingly,

$$
\ddot{X}_{b}=\ddot{u}_{b}
$$

If the supported mass matrix is sufficiently large and a sufficiently large number has been assigned to the supporting mass matrix of the general mass matrices, then the ground acceleration in the supporting points can be equal to the seismic response acceleration. As a result, Eq. (2) can be easily solved.

Under uniform-tuning non-stationary excitation, the ground supporting force is expressed as

$$
P_{b}=M_{b b} \cdot \ddot{X}_{b}=M_{b b} \cdot G(t) \cdot \ddot{u}_{b}(t)
$$

where $\mathrm{G}(\mathrm{t})$ is the determining time-tuning function and $\ddot{\boldsymbol{u}}_{\mathrm{b}}(t)$ is a stationary random process.

$$
\begin{aligned}
& \boldsymbol{G}(t)=\operatorname{diag}\left[\mathrm{G}_{1 \mathrm{x}}(t), \mathrm{G}_{1 \mathrm{y}}(t), \mathrm{G}_{1 \mathrm{z}}(t), \mathrm{G}_{2 \mathrm{x}}(t), \mathrm{G}_{2 \mathrm{y}}(t),\right. \\
& \left.\mathrm{G}_{2 \mathrm{z}}(t), \cdots, \mathrm{G}_{\mathrm{mx}}(t), \mathrm{G}_{\mathrm{my}}(t), \mathrm{G}_{\mathrm{mz}}(t)\right] \\
& \ddot{\boldsymbol{u}}_{\mathrm{b}}(t)=\left[\ddot{u}_{1 \mathrm{x}} \ddot{u}_{1 \mathrm{y}} \ddot{u}_{1 \mathrm{z}} \ddot{u}_{2 \mathrm{x}} \ddot{u}_{2 \mathrm{y}} \ddot{u}_{2 \mathrm{z}} \cdots \ddot{u}_{\mathrm{mx}} \ddot{u}_{\mathrm{my}} \ddot{u}_{\mathrm{mz}}\right]
\end{aligned}
$$

In practical engineering, the ground motion time-tuning function is presumed to be consistent, that is,

$$
G_{m x}(t)=G_{m y}(t)=G_{m z}(t)=G_{g}(t)
$$

\subsection{Modeling of tri-directional spatially varying ground} motions and determination of pseudo excitation forces

The cross-power spectral density function of tri-directional, non-stationary, spatially varying ground motions at $\mathrm{m}$ spatial points can be expressed as

$$
\left[\boldsymbol{S}_{0}(\mathrm{i} \omega, t)\right]=\left[\begin{array}{ccccc}
\boldsymbol{S}_{11}(\mathrm{i} \omega, t) & \boldsymbol{S}_{12}(\mathrm{i} \omega, t) & \boldsymbol{S}_{13}(\mathrm{i} \omega, t) & \mathrm{L} & \boldsymbol{S}_{1 \mathrm{~m}}(\mathrm{i} \omega, t) \\
\boldsymbol{S}_{21}(\mathrm{i} \omega, t) & \boldsymbol{S}_{22}(\mathrm{i} \omega, t) & \boldsymbol{S}_{23}(\mathrm{i} \omega, t) & \mathrm{L} & \boldsymbol{S}_{2 \mathrm{~m}}(\mathrm{i} \omega, t) \\
\boldsymbol{S}_{31}(\mathrm{i} \omega, t) & \boldsymbol{S}_{32}(\mathrm{i} \omega, t) & \boldsymbol{S}_{33}(\mathrm{i} \omega, t) & \mathrm{L} & \boldsymbol{S}_{3 \mathrm{~m}}(\mathrm{i} \omega, t) \\
\mathrm{M} & \mathrm{M} & \mathrm{M} & 0 & \mathrm{M} \\
\boldsymbol{S}_{\mathrm{m} 1}(\mathrm{i} \omega, t) & \boldsymbol{S}_{\mathrm{m} 2}(\mathrm{i} \omega, t) & \boldsymbol{S}_{\mathrm{m} 3}(\mathrm{i} \omega, t) & \mathrm{L} & \boldsymbol{S}_{\mathrm{mm}}(\mathrm{i} \omega, t)
\end{array}\right]
$$

where each sub-matrix element $S_{k l}(i w, t)$ is a $3 \times 3$ matrix corresponding to two horizontal (x,y) and one vertical (z) components of tri-directional ground motions; it is given by

$$
\boldsymbol{S}_{\mathrm{kl}}(\mathrm{i} \omega, t)=\left[\begin{array}{lll}
\mathrm{S}_{\mathrm{klxx}}(\mathrm{i} \omega, t) & \mathrm{S}_{\mathrm{klxy}}(\mathrm{i} \omega, t) & \mathrm{S}_{\mathrm{klxz}}(\mathrm{i} \omega, t) \\
\mathrm{S}_{\mathrm{klyx}}(\mathrm{i} \omega, t) & \mathrm{S}_{\mathrm{klyy}}(\mathrm{i} \omega, t) & \mathrm{S}_{\mathrm{klyz}}(\mathrm{i} \omega, t) \\
\mathrm{S}_{\mathrm{klzx}}(\mathrm{i} \omega, t) & \mathrm{S}_{\mathrm{klzy}}(\mathrm{i} \omega, t) & \mathrm{S}_{\mathrm{klzz}}(\mathrm{i} \omega, t)
\end{array}\right]
$$

The power spectral density functions of the horizontal components of tri-directional ground motions are assumed to be similar, and the correlation coefficients between the horizontal and vertical components are assumed to be 0.6. 


$$
\begin{aligned}
& \mathrm{S}_{\mathrm{klxx}}(\mathrm{i} \omega, t)=\mathrm{S}_{\mathrm{klyy}}(\mathrm{i} \omega, t)=\mathrm{S}_{\mathrm{klxy}}(\mathrm{i} \omega, t)=\mathrm{S}_{\mathrm{klyx}}(\mathrm{i} \omega, t) \\
& \mathrm{S}_{\mathrm{klxz}}(\mathrm{i} \omega, t)=\mathrm{S}_{\mathrm{klyz}}(\mathrm{i} \omega, t)=0.6 \sqrt{\mathrm{S}_{\mathrm{klxx}}(\mathrm{i} \omega, t) \mathrm{S}_{\mathrm{klzz}}(\mathrm{i} \omega, t)} \\
& =0.6 \sqrt{\mathrm{S}_{\mathrm{klyy}}(\mathrm{i} \omega, t) \mathrm{S}_{\mathrm{klzz}}(\mathrm{i} \omega, t)}
\end{aligned}
$$

According to the theory of the evolutionary power spectrum for non-stationary random processes, the elements of the cross-spectral density matrix are defined as

$\mathrm{S}_{\mathrm{klkx}}(\mathrm{i} \omega, t)=\left\{\begin{array}{c}\mathrm{G}_{\mathrm{kkxx}}(t) \mathrm{G}_{\mathrm{llkx}}(t) \sqrt{\mathrm{S}_{\mathrm{kkxx}}(\mathrm{i} \omega, t) \mathrm{S}_{\mathrm{llkx}}(\mathrm{i} \omega, t)} \rho_{\mathrm{klxx}}(\mathrm{i} \omega), \\ \left|\mathrm{G}_{\mathrm{kkkx}}(t)\right|^{2} \mathrm{~S}_{\mathrm{kkkx}}(\mathrm{i} \omega),\end{array}\right.$

where $G_{k k x x}(t)$ and $S_{k k x x}(i \omega)$ are the uniformly modulating function and auto-power spectral density function of the $\mathrm{x}$ component of tri-directional ground motions at the kth spatial support, respectively; $\rho_{k l x x}(i \omega)$ are the uniformly modulating function and auto-power spectral density function of the $\mathrm{x}$ component of tri-directional ground motions at the kth spatial support, respectively.

The power spectral density matrix $\boldsymbol{S}_{0}(i \omega, t)$ of tridirectional non-stationary spatially varying ground motions can be decomposed as

$$
S_{0}(i \omega, t)=P^{*} P^{T}=[G(t)][V] *\left[S_{C P}\right]\left\{q_{0}\right\}\left\{q_{0}\right\}^{T}\left[S_{C P}\right][V]^{T}[G(t)]^{T}
$$

where $P$ is the pseudo-force matrix with a dimension of $3 m \times r, r$ is the rank of matrix $S_{0}(i \omega, t)$, and superscripts * and $T$ refer to the complex conjugate and transpose, respectively.

The vector of $S_{\mathrm{CP}}$ has a dimension of $3 \mathrm{~m} \times 3 \mathrm{~m}$ and is given by

$$
\begin{aligned}
& {\left[S_{C P}(w)\right]=\operatorname{diag}\left[\sqrt{S_{C P 1 x}}(w), \sqrt{S_{C P 1 y}}(w), \sqrt{S_{C P 1 z}}(w),\right.} \\
& \left.\mathrm{K}, \sqrt{S_{C P m x}}(w), \sqrt{S_{C P m y}}(w), \sqrt{S_{C P m z}}(w)\right]
\end{aligned}
$$

where $\boldsymbol{S}_{\mathrm{CPmx}}(\omega)$ the denotes power spectral density function of the $\mathrm{x}$ component of ground motions in the mth spatial support. by

The vector of $\mathrm{V}$ with a dimension of $3 m \times 3 m$ is given

$$
\begin{aligned}
& {[V]=\operatorname{diag}\left[e^{-i \omega T_{1 x}}, e^{-i \omega T_{1 y}}, e^{-i \omega T_{1 z}}, e^{-i \omega T_{2 x}},\right.} \\
& \left.e^{-i \omega T_{2 y}}, e^{-i \omega T_{2 z}}, \mathrm{~L}, e^{-i \omega T_{m x}}, e^{-i \omega T_{m y}}, e^{-i \omega T_{m z}}\right]
\end{aligned}
$$

where $T_{m x}$ represents the time at which the x component of ground motions propagate to the $\mathrm{mth}$ support. The wavepassage effect is reflected in vector $V$.

The vector of $G(t)$ has a dimension of $3 m \times 3 m$ and is given by

$$
\begin{aligned}
& G(t)=\operatorname{diag}\left[G_{1 x}(t), G_{1 y}(t), G_{1 z}(t), G_{2 x}(t),\right. \\
& \left.G_{2 y}(t), G_{2 z}(t), \mathrm{L}, G_{m x}(t), G_{m y}(t), G_{m z}(t)\right]
\end{aligned}
$$

where $\mathrm{G}_{\mathrm{mx}}(t)$ is a modulating function of the mth support in $\mathrm{x}$ direction. Owing to Eq. (10), Eq. (19) can be simplified as

$$
\begin{aligned}
& G(t)=\operatorname{diag}[G(t), G(t), G(t), G(t), G(t), \\
& \mathrm{G}(t), \mathrm{L}, \mathrm{G}(t), \mathrm{G}(t), \mathrm{G}(t)]
\end{aligned}
$$

$[R]$ is the lagged coherence matrix provided by

$$
[R]=\left\{q_{0}\right\}\left\{q_{0}\right\}^{T}=\left[\begin{array}{cccc}
1 & \left|\rho_{12}\right| & \mathrm{L} & \left|\rho_{1 N}\right| \\
\left|\rho_{21}\right| & 1 & \mathrm{~L} & \left|\rho_{2 N}\right| \\
\mathrm{M} & \mathrm{M} & 0 & \mathrm{M} \\
\left|\rho_{N 1}\right| & \left|\rho_{N 2}\right| & \mathrm{L} & 1
\end{array}\right]
$$

where $N=3 \mathrm{~m}$ and $[R]$ is a definite or semi-definite symmetric matrix. The rank of $[R]$ is greater than 1 and can be decomposed as the summation of non-zero eigenvalues $\{\varphi\}_{\mathrm{j}}(j=1,2, \ldots, r, r \leq 3 m)$, which is given by

$$
\left\{\begin{array}{c}
{[R]\{\varphi\}_{j}=\alpha_{j}\{\varphi\}_{j}} \\
\{\varphi\}_{j}{ }_{j}^{T}\{\varphi\}_{j}^{*}=\delta_{i j}(j=1,2, \mathrm{~L}, r) \\
{[R]=\sum_{j=1}^{r} \alpha_{j}\{\varphi\}_{j}{ }_{j}^{*}\{\varphi\}_{j}{ }^{T}}
\end{array}\right.
$$

The pseudo-excitations can be obtained based on the coherence level of the spatially varying ground motions.

$P=\left\{\begin{array}{c}{[G(t)][V]\left\{q_{0}\right\} \sqrt{S_{C P}(\omega)} e^{i \omega t}} \\ {[G(t)][V]\{\varphi\}_{j} \sqrt{\alpha_{j} S_{C P}(\omega)} e^{i \omega t}} \\ {[G(t)][V] \sqrt{S_{C P}(\omega)} e^{i \omega t}}\end{array}\right.$

Incorporating the second item in Eq. (23) into Eq. (7) results in

$P_{b}=M_{b} \cdot P$

The pseudo responses can then be derived after obtaining the pseudo-excitations at different coherence levels.

The pseudo response of absolute displacement $y / \frac{\rho}{a j}$, with respect to the $\mathrm{j}^{\text {th }}$ eigenvalue can be obtained as follows:

$\tilde{y}_{a j}=\sqrt{\alpha_{j} S_{C P}(\omega)} I_{j}(t)$

where

$I_{j}(t)=\int_{0}^{t} h(t-\tau) G(\tau) V\{\varphi\}_{j} e^{i \omega \tau} d \tau$

The power spectral density of the pseudo response of absolute displacement under partially coherent tri-directional non-stationary ground motions can be expressed as

$$
S_{X_{S} X_{S}}(\omega, t)=\sum_{j=1}^{r} \tilde{y}_{a j}^{*} \tilde{y}_{a j}^{T}=\sum_{j=1}^{r} I_{j}^{*} I_{j}^{T} \alpha_{j} S_{C P}(\omega)
$$

Similarly, the power spectral densities of the pseudo response of absolute displacement $\tilde{y}_{a}$ under fully coherent and completely incoherent spatial ground motions can be obtained, as shown below. 
Fully coherent:

$$
S_{X_{S} X_{S}}(\omega, t)=\tilde{y}_{a}^{*} \tilde{y}_{a}^{T}=I_{0}^{*} I_{0}^{T} S_{C P}(\omega)
$$

where

$$
I_{0}(t)=\int_{0}^{t} h(t-\tau) G(\tau) V\left\{q_{0}\right\} e^{i \omega \tau} d \tau
$$

Completely incoherent:

$$
S_{X_{S} X_{S}}(\omega, t)=\tilde{y}_{a}^{*} \tilde{y}_{a}^{T}=I^{*} I^{T} S_{C P}(\omega)
$$

where

$$
I(t)=\int_{0}^{t} h(t-\tau) G(\tau) V e^{i \omega \tau} d \tau
$$

According to Eqs. (26), (29), and (31), transient analysis and calculation need to be completed at each frequency point Although the direct-solving pseudo-excitation method saves time compared with traditional stochastic vibration theory, more calculations of transient analysis are required when the discrete points are excessive; thus, the method is more time consuming than the new method proposed in this study. With the improved high-precision integration method and its realization in a general finite element software, the structural response can be determined at any node if two transient analyses are ready. This scenario significantly reduces the computation time. Reduced computation time is convenient for engineering applications. Matrix manipulation in the first block row of Eq. (1) to construct a pseudo-excitation force results in

$$
M_{s s} \ddot{\tilde{X}}_{s}+C_{s s} \dot{\tilde{X}}_{s}+K_{s s} \tilde{X}_{s}=-\tilde{Y}(\omega, t)
$$

where

$$
\tilde{Y}(\omega, t)=M_{b b} \cdot P_{b}=M_{b b} \cdot P_{b}(\omega, t)
$$

$\tilde{Y}(\omega, t)$ is the non-stationary stochastic excitation time history under the determined frequency points.

The dynamic equations under the determined frequency points in Eq. (32) can be written as equations of state [15$16]$.

$$
\dot{V}=H V+F(\omega, t)
$$

$V=\left\{\begin{array}{c}\tilde{X}_{s} \\ \dot{\tilde{X}}_{s}\end{array}\right\}, F(\omega, t)=-W \tilde{Y}(\omega, t), H=\left[\begin{array}{cc}0 & I \\ -M^{-1} K & -M^{-1} C\end{array}\right]$,

$W=\left[\begin{array}{c}0 \\ M^{-1}\end{array}\right]$

The general solution of Eq. (32) is

$$
V(t)=e^{H t} V(0)+\int_{0}^{t} e^{H(t-\tau)} F(\omega, \tau) d \tau
$$

where $e^{H t}$ is the exponential matrix, a discrete integral of Eq. (36). By setting the time step to $\Delta t$ and using the recursive method, the response at time $\mathrm{t}_{\mathrm{i}}, \boldsymbol{V}\left(t_{i}\right)=\boldsymbol{V}_{i}$, can be expressed with the response at time $\mathrm{t}_{\mathrm{i}-1}, \boldsymbol{V}\left(t_{i-1}\right)=\boldsymbol{V}_{i-1}$, by the following:

$$
V_{i}=e^{H t_{i}} V(0)+\int_{0}^{t_{i}} e^{H\left(t_{i}-\tau\right)} F(\omega, \tau) d \tau=T \cdot V_{i-1}+\int_{t_{i-1}}^{t_{i}} e^{H\left(t_{i}-\tau\right)} F(\omega, \tau) d \tau^{(37)}
$$

where $\boldsymbol{T}=\mathrm{e}^{H \Delta t}$ is the exponential matrix. According to the precise algorithm in Reference [9], $T$ can be rewritten as

$$
T=e^{H \Delta t}=\left[e^{H \Delta t / m}\right]^{m}
$$

By setting $\tau=\Delta t / m$ when $m=2^{N}$ is large and $\Delta \tau$ is small, a Taylor series expansion is provided by

$$
T(\tau)=\left[e^{H \tau}\right]^{m} \cong\left[I+H \tau+\mathrm{L}+\frac{(H \tau)^{4}}{4 !}\right]^{m} \equiv\left[I+T_{a 0}\right]^{m}
$$

When $N=20$, the Taylor series truncation error is noticeably smaller than the computer rounding error. In actuality, truncation would not result in a numerical error. Therefore, the computer's exact solutions are provided because of the calculation precision of $\boldsymbol{T}(\tau)$.

$$
\left[I+T_{a i}\right] \equiv\left[I+T_{a, i-1}\right]^{2}=\left[I+2 T_{a, i-1}+T_{a, i-1} \times T_{a, i-1}\right](i=1,2, \mathrm{~L}, N)
$$

Hence, if we analogize in turn,

$$
\left[I+T_{a N}\right] \equiv\left[I+T_{a, N-1}\right]^{2}=\mathrm{L}=\left[I+T_{a 0}\right]^{m}=T(\tau)
$$

In each operation, unit matrix $[I]$ is not involved in the calculation because $T_{a i}$ is small and would become the mantissa when added to unit matrix [I]. As a result, it is ignored in the computer rounding operation. To avoid this situation, the following recursive solution is used.

$$
\left[T_{a i}\right]=2\left[T_{a, i-l}\right]+\left[T_{a, i-l}\right]\left[T_{a, i-l}\right](i=1,2, \ldots, N)
$$

In each integration step $\left(t_{i-1}, t_{i}\right)$, the load is assumed to change linearly. Non-stationary pseudo force $F(\omega, t)$ can be discretized as stochastic variables $F_{0}, F_{1}, F_{2}, \cdots, F_{k}$ at time $t_{0}, t_{1}, t_{2}, \ldots, t_{\mathrm{k}}$, respectively, by the following:

$$
F(\omega, t)=r_{0}+r_{1}\left(\tau-t_{i-1}\right)
$$

where $r_{0}, r_{1}$ is a time-invariant vector.

Inserting Eq. (43) into Eq. (37) results in

$$
\begin{aligned}
& V_{i}=T \cdot V_{i-1}+\int_{t_{i-1}}^{t_{i}} e^{H\left(t_{i}-\tau\right)}\left(r_{0}+r_{1}\left(\tau-t_{i-1}\right)\right) d \tau \\
& =T \cdot V_{i-1}-H^{-1}\left[H^{-1}\left(F_{i}-F_{i-1}\right) / \Delta t+F_{i}\right]+ \\
& T H^{-1}\left[H^{-1}\left(F_{i}-F_{i-1}\right) / \Delta t+F_{i-1}\right](i=1,2, \mathrm{~L}, k)
\end{aligned}
$$

where $\boldsymbol{H}^{-1}$ can be derived according to Eq. (35). 
$H^{-1}=\frac{H^{*}}{|H|}=\frac{\left[\begin{array}{cc}-M^{-1} C & -I \\ M^{-1} K & 0\end{array}\right]}{M^{-1} K}=\left[\begin{array}{cc}-K^{-1} C & -M K^{-1} \\ I & 0\end{array}\right]$

Eq. (44) is further written as follows:

$$
\begin{aligned}
& V_{i}=T \cdot V_{i-1}-H^{-1}\left[H^{-1}\left(F_{i}-F_{i-1}\right) / \Delta t+F_{i}\right]+T H^{-1}\left[H^{-1}\left(F_{i}-F_{i-1}\right) / \Delta t+F_{i-1}\right] \\
& =T \cdot V_{i-1}+\left[(I-T)\left(H^{-2} / \Delta t\right)+T H^{-1}\right] F_{i-1}+\left[(T-I)\left(H^{-2} / \Delta t\right)-H^{-1}\right] F_{i}
\end{aligned}
$$

Set

$$
S_{1}=(I-T)\left(H^{-2} / \Delta t\right)+T H^{-1}, S_{2}=(T-I)\left(H^{-2} / \Delta t\right)-H^{-1}
$$

where $H^{-2}=H^{-1} \cdot H^{-1}$. Eq. (66) can be simplified as

$$
V_{i}=T \cdot V_{i-1}+S_{1} F_{i-1}+S_{2} F_{i}(i=1,2, \mathrm{~L}, k)
$$

At each fixed frequency point, $\boldsymbol{V}_{0}=\mathbf{0}$. Through Eq. (48), the response at each moment can be expressed as

$$
\left\{\begin{array}{c}
V_{1}=S_{1} F_{0}+S_{2} F_{1}(i=1) \\
V_{i}=T^{i-1} S_{1} F_{0}+T^{i-2} S_{3} F_{1}+\mathrm{L}+T^{0} S_{3} F_{i-1} \\
+S_{2} F_{i}(2>>>>k)
\end{array}\right.
$$

where

$$
S_{3}=T S_{2}+S_{1}
$$

$A_{i, 0}, A_{i, 1}, \cdots, A_{i, I}$ represent the coefficients in front of $F_{0}, F_{1}, \cdots, F_{i}$, respectively, and the $i$ in $A_{i, i}$ stands for time $t_{i}$. The second $i$ denotes the $i$ th load discrete point location. Eq. (44) can be expressed as:

$$
V_{i}=A_{i, 0} F_{0}+A_{i, 1} F_{1}+\cdots+A_{i, i-1} F_{i-1}+A_{i, i} F_{i}(i=1,2, \mathrm{~L}, k)
$$

where $A_{i, 0}, A_{i, 1}, \cdots, A_{i, I}$ is connected to the structure itself but not to the external load, reflecting the inherent properties of the structure under external load excitations [15]. The calculation process of $A_{i, i}$ is recursive, and the coefficient of $V_{i-1}\left(A_{i-1,0}, A_{i-1,1}, \cdots, A_{i-1, i-1}\right)$ is used to deduce the next coefficient, which is

$$
\left\{\begin{array} { c } 
{ A _ { 1 , 0 } = S _ { 1 } } \\
{ A _ { 1 , 0 } = T A _ { 1 , 0 } }
\end{array} \left\{\begin{array} { c } 
{ A _ { i , 0 } = T A _ { i - 1 , 0 } } \\
{ A _ { 1 1 } = S _ { 2 } } \\
{ A _ { 2 1 } = S _ { 3 } }
\end{array} \left\{\begin{array}{l}
A_{i, 1}=T A_{i-1,1} \\
A_{2,2}=A_{11}(2 \ll<j<i, 3 \ll i<<k) \\
A_{i, j}=A_{i-1, j-1}
\end{array}\right.\right.\right.
$$

The result of substituting Eq. (52) into Eq. (51) written in matrix form is [16-17]

$$
\left[\begin{array}{cccccccc}
0 & & & & & & & \\
A_{1,0} & A_{1,1} & & & & & & \\
A_{2,0} & A_{2,1} & A_{1,1} & & & & & \\
A_{3,0} & A_{3,1} & A_{2,1} & A_{1,1} & & & & \\
\mathrm{M} & \mathrm{M} & \mathrm{M} & \mathrm{M} & 0 & & & \\
A_{k-2,0} & A_{k-2,1} & A_{k-3,1} & A_{k-4,1} & \mathrm{~L} & A_{1,1} & & \\
A_{k-1,0} & A_{k-1,1} & A_{k-2,1} & A_{k-3,1} & \mathrm{~L} & A_{2,1} & A_{1,1} & \\
A_{k, 0} & A_{k, 1} & A_{k-1,1} & A_{k-2,1} & \mathrm{~L} & A_{3,1} & A_{2,1} & A_{1,1}
\end{array}\right]\left[\begin{array}{c}
F_{0}\left(t_{0}\right) \\
F_{1}\left(t_{1}\right) \\
F_{2}\left(t_{2}\right) \\
F_{3}\left(t_{3}\right) \\
\mathrm{M} \\
F_{k-2}\left(t_{k-2}\right) \\
F_{k-1}\left(t_{k-1}\right) \\
F_{k}\left(t_{k}\right)
\end{array}\right]=\left[\begin{array}{c}
V_{0}\left(t_{0}\right) \\
V_{1}\left(t_{1}\right) \\
V_{2}\left(t_{2}\right) \\
V_{3}\left(t_{3}\right) \\
\mathrm{M} \\
V_{k-2}\left(t_{k-2}\right) \\
V_{k-1}\left(t_{k-1}\right) \\
V_{k}\left(t_{k}\right)
\end{array}\right]
$$

According to Eq. (51), all the other elements in the coefficient matrix are repeated in the first and second columns. In other words, as long as the elements in the first and second columns are obtained, the entire coefficient matrix can be determined. Setting $F 0=1$ and $F k=0(k>0)$ allows the first column elements of the coefficient matrix to be derived through transient analysis. Similarly, setting $\mathrm{F} 0=0, \mathrm{~F} 1=1$, and $\mathrm{Fk}=1(\mathrm{k}>2)$ allows the second column elements of the coefficient matrix to be obtained.

Under a non-stationary time history load, the response at any frequency point can be derived according to Eq. (53) once the coefficient matrix has been obtained. Under multidimensional, multi-support, and non-stationary seismic excitation loads, when absolute displacement is used to solve directly, each incentive point of the structure needs to be excited in three directions. Then, the coefficient matrix A of the required structural response at key points can be obtained. After multiplying and superimposing the excitation time history in every direction and the corresponding coefficient matrix $\mathrm{A}$, the structural response at fixed frequency points can be derived. The structural response over the entire frequency range can be determined by substituting the excitation process at all frequency points. Time history analyses under multi-dimensional and multisupport frequency points could thus be avoided, and computation time would be reduced.

\section{Results Analysis and Discussion}

\subsection{Example of the SDOF Structure}

This study is limited to the linear elastic range and the use of the finite element method to calculate a structural response. The superposition principle still applies. Consequently, verifying the correctness of the method requires an SDOF system. To verify the calculation accuracy of the proposed method under non-stationary pseudo excitation in ANSYS, we use analytical solutions and absolute displacement under the acceleration power spectrum Clough-Penzien to solve directly based on the SDOF system provided by Caughey.

The mathematic statement is [18]

$$
\ddot{y}+2 \xi \omega_{0}+\omega_{0}^{2} y=f(x)=g(x) x(t)
$$

$y(0)=\dot{y}(0)=0$

where

$$
g(t)=\left\{\begin{array}{cc}
\left(t / t_{1}\right)^{2} & 0 \leq t \leq t_{1} \\
1 & t_{1} \leq t \leq t_{2} \\
\mathrm{e}^{-c\left(t-t_{2}\right)} & t \geq t_{2}
\end{array}\right.
$$

According to literature [2], $t_{1}=7.1, t_{2}=19.5$, and $c=0.16$.

$$
S(\omega)=\frac{1+4 \xi_{\mathrm{g}}^{2}\left(\omega / \omega_{\mathrm{g}}\right)^{2}}{\left[1-\left(\omega / \omega_{\mathrm{g}}\right)^{2}\right]^{2}+4 \xi_{\mathrm{g}}^{2}\left(\omega / \omega_{\mathrm{g}}\right)^{2}} \cdot \frac{\omega^{4}}{\left(\omega^{2}-\omega_{\mathrm{f}}^{2}\right)^{2}+4 \xi_{\mathrm{f}}^{2} \omega_{\mathrm{f}}^{2} \omega^{2}} \cdot S^{*}
$$

where the spectrum intensity factor $\mathrm{S}^{*}=1\left(\mathrm{~m}^{2} / \mathrm{s}^{3}\right)$, the field parameters $\omega_{g}=15.0(\mathrm{rad} / \mathrm{s}), \xi_{\mathrm{g}}=0.6, \omega_{\mathrm{f}}=1.5(\mathrm{rad} / \mathrm{s}), \xi_{\mathrm{f}}=0.6$ when $m=1.0(\mathrm{Kg}), K=1.0(\mathrm{~N} / \mathrm{m})$, and $\xi=0.05$. The variance of the response is presented in Fig. 1. 


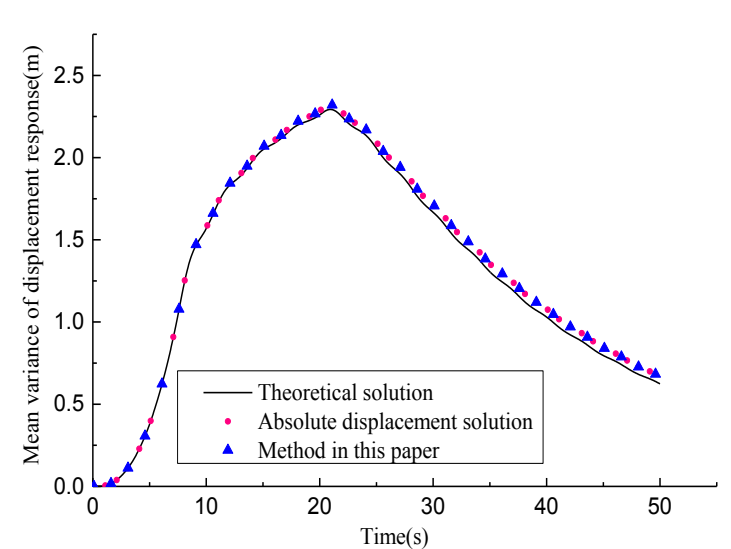

Fig. 1. Mean variance of the displacement response

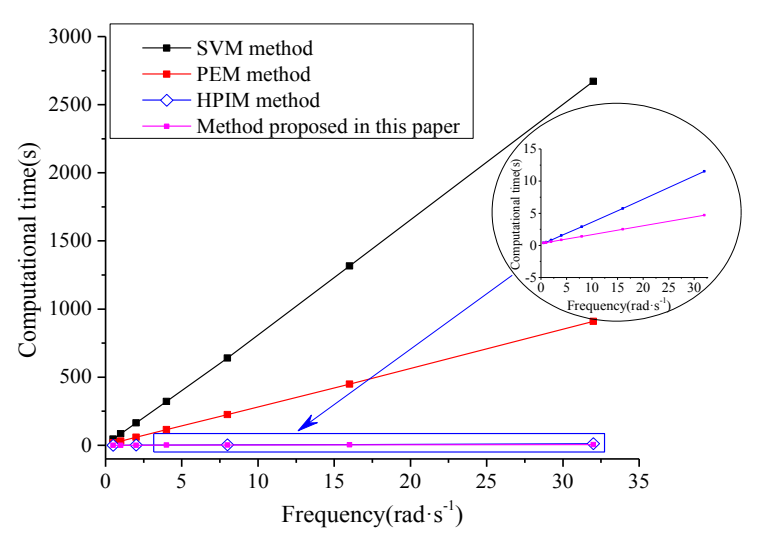

Fig. 2. Comparison of computational efficiency

As shown in Fig. 1, the result of the method proposed in this study (absolute displacement solution combined with a high-precision integration method) agrees with those of the theoretical calculation and the absolute displacement solution. In addition, the proposed method implements highly precise calculations.

The selection of the SDOF structure for an efficient comparison is based on two considerations. The first is that the structural response under non-stationary seismic excitation involves a large amount of calculation difficulty and is likely impractical. Second, the general structure can be converted to an SDOF system through a modesuperposition method over the elastic range. Moreover, the SDOF system is the foundation of the MDOF system. Setting the SDOF system as an example through the new method is highly efficient. The traditional stochastic vibration method, pseudo excitation method, pseudo excitation and high-precision integration method, and IHPIM methods are selected in this study to compare computer efficiency. Additionally, the Duhamel integral method was adopted for a more convenient comparison. The integral step is 0.01 (s), the seismic calculation time is 30 (s), and the time step is 0.05 (s). The other parameters are consistent with those for the SDOF system in Section 4.1, and the computational results are as shown in Fig. 2.

Table 1 shows the calculation time with the SDOF system at any frequency point (including the post-processing time). As shown in Fig. 2 and Table 1, the method proposed in this study has the highest efficiency among all four methods; this preponderance becomes increasingly apparent as the computing frequency points increase. SVM (the traditional stochastic vibration method) is difficult to apply in practical engineering because of its long calculation time. The efficiency of PEM (the pseudo-excitation method) is 3 times as high as that of SVM because it avoids the multiple integrals. For a large and complex structure, seismic analysis still needs improvement. The efficiency of HPIM (pseudoexcitation method and high-precision integration method) improves by 115 times because of the introduced highprecision integration. The efficiency of I-HPIM, which is combined with the absolute displacement direct solution (the method proposed in this study), improves 120 times on the basis of SVM. As a result, the proposed method is convenient for multi-dimensional and multi-support seismic excitation simulations.

Table 1. Comparison of the calculation time of the four methods

\begin{tabular}{l|l|l|l|l}
\hline Frequency & SVM & PEM & HPIM & I-HPIM \\
\hline 0.5 & 45.72 & 17.83 & 0.39 & 0.37 \\
1 & 84.25 & 30.77 & 0.48 & 0.42 \\
2 & 163.51 & 60.06 & 0.81 & 0.55 \\
4 & 322.02 & 115.81 & 1.54 & 0.85 \\
8 & 639.49 & 224.73 & 2.91 & 1.39 \\
16 & 1315.80 & 449.39 & 5.73 & 2.50 \\
32 & 2671.80 & 909.52 & 11.50 & 4.68 \\
\hline Note: The frequency unit is rad/s, and the time unit is s. SVM: \\
traditional stochastic vibration method, PEM: pseudo excitation \\
method, HPIM: pseudo excitation method and high-precision \\
integration method, I-HPIM: the method proposed in this study.
\end{tabular}

\subsection{Finite Element Mode and Parameter Description}

A long-span high-pier continuous rigid frame bridge is employed in this study. The 3D FE model of the bridge is presented in Fig. 3. Fixed boundary conditions are applied to the bottoms of Pier \#1 to Pier \#5, and the vertical (Z), transverse $(\mathrm{Y})$, and rotational DoFs with respect to $\mathrm{Z}$ (Rotz) and $\mathrm{X}$ (Rotx) directions are fixed in the north and south abutments of the railway bridge. All the DoFs of Pier \#1, Pier \#2, and Pier \#4 (the DoFs in the connection area of the piers and main girder) are coupled with the main girder's corresponding DoFs, whereas the longitudinal DoFs (X) of Pier \#3, Pier \#5, and the main girder in their connection parts are released.

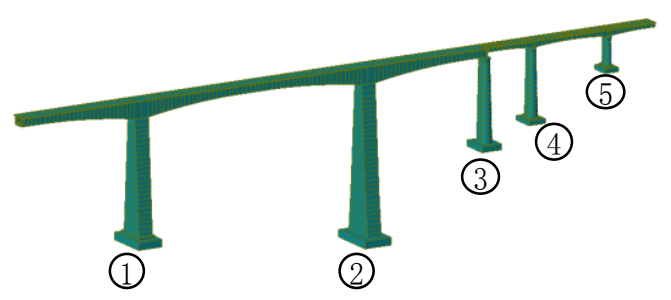

Fig. 3. 3D FE model

The Clough-Penzien power spectrum is used. Parametric spectral intensity factor $\mathrm{S}^{*}=0.00177\left(\mathrm{~m}^{2} / \mathrm{s}^{3}\right)$, field parameters $\omega_{\mathrm{g}}=15.0(\mathrm{rad} / \mathrm{s}), \quad \xi_{\mathrm{g}}=0.6, \omega_{\mathrm{f}}=1.5(\mathrm{rad} / \mathrm{s}), \quad$ and $\xi_{\mathrm{f}}=0.6$. The modulating functions and parameters in Eq. (56) are adopted.

The purpose of this study is to introduce calculation theory and the correctness of quick calculations and highly efficient verifications. However, the practical application of these calculations will be discussed in subsequent papers. The relative displacement response time-varying variance of the expansion joints in the top two main girders of pier \#2 is calculated with the four methods. The results are shown in Figs. 4 to 6 . A solid foundation is established to design wide 
expansion joints for high-pier bridges and thus prevent beams from colliding with each other under earthquake stress..

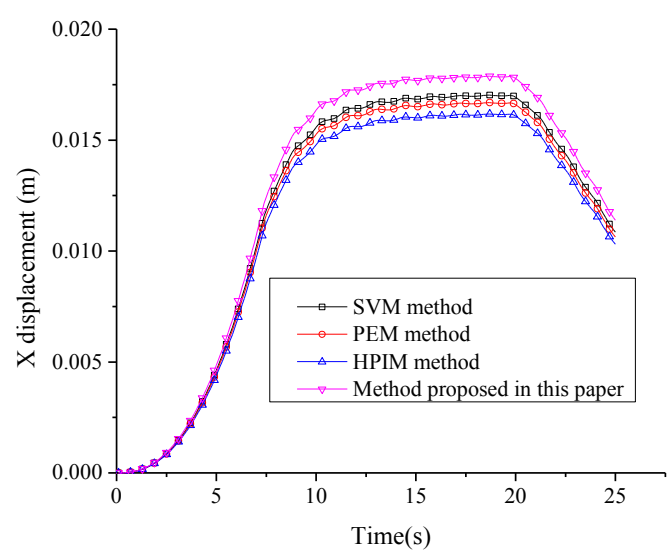

Fig. 4. Relative displacement of expansion joints in hard soil conditions

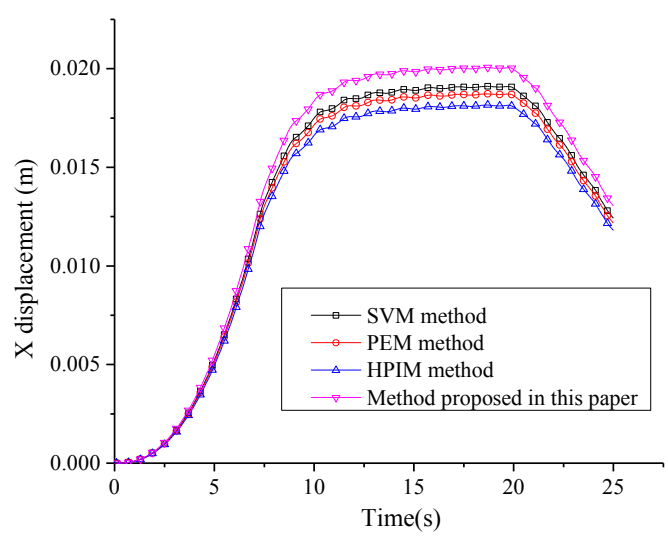

Fig. 5. Relative displacement of expansion joints in medium soil conditions

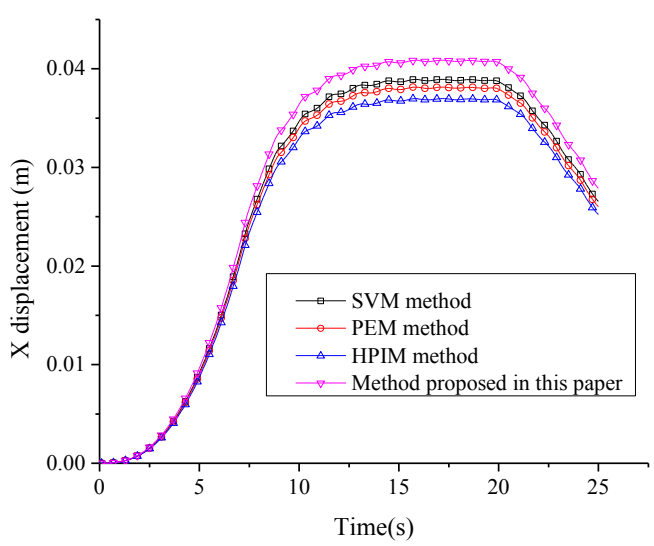

Fig. 6. Relative displacement of expansion joints in soft soil conditions
As shown in Figs. 4 to 6 , the relative displacement responses of the four methods are in good agreement. When compared with the traditional stochastic vibration method, the difference does not exceed 5\%. The methods proposed in this study are conservative because the damping coefficient is proportional to the absolute displacement, and the traditional damping coefficient is proportional to the relative displacement. Among the three soil types (hard, medium, and soft), the soft soil site has the greatest relative displacement response. The influence of a soft soil site on the structural response should be considered with expansion joint width design largely because high piers lead to low vibration frequency, which is close to the predominant frequency.

\section{Conclusions}

To improve the computational efficiency of a complex structural dynamic response under non-stationary seismic excitation, a fast simulation method was developed and implemented with ANSYS software. The following conclusions were obtained.

(1) The proposed method fully takes considers real ground motions, so calculating the dynamic response of a large-span complex structure in consideration of the spatial effects of ground motion, non-stationarity, and multi-dimension is easy. Additionally, efficient calculation and analysis methods are provided for the seismic design code.

(2) The improved high-precision integration method allows for the time history analysis of $\mathrm{N}$ (number of discrete frequency points) reduced to a time history analysis of $2 \times 3 \times \mathrm{m}$ ( $\mathrm{m}$ is the number of supporting nodes). As a result, the computational efficiency is improved rapidly.

(3) The proposed method is based on the general finite element software ANSYS so that engineers can easily master it. The method thus contributes to the application and promotion of seismic analysis in practical engineering.

The proposed theoretical approach was implemented and verified in the general finite element platform to make it readily applied in stochastic seismic analysis of several complex and significant structures.

\section{Acknowledgements}

The authors are grateful for the supports provided by the National Natural Science Funds (Grant No. 51308465) and the Fundamental Research Funds for the Central Universities (Grant No. 682014CX004EM).

\section{References}

1. Heredia - Zavoni, Ernesto, Sandra Santa - Cruz, and Francisco L. "Modal response analysis of multi - support structures using a random vibration approach". Earthquake Engineering \& Structural Dynamics, 44(13), 2015, pp.2241-2260.
2. Lin J H, Zhao $\mathrm{Y}$, Zhang $\mathrm{Y} \mathrm{H}$. "Accurate and Highly Efficient Algorithms for Structural Stationary/ Non-stationary random responses". Computer Methods in Applied Mechanics and Engineering. 191(1-2), 2001, pp.103-111. 
Rui Kang, Jin Zhang, Liu-Xi Zhou, Shi-Qiang Qin, Kai-Feng Zheng and De-Yi Zhang/

Journal of Engineering Science and Technology Review 9 (3) (2016) 106 - 113

3. Ma, C. F., Y. H. Zhang, P. Tan, David Kennedy, F. W. Williams, and F. L. Zhou. "Non-stationary seismic response analysis of baseisolated buildings with many hysteretic devices". Computers \& Structures. 123, 2013, pp.39-47.

4. Settineri D. "An effective method for the evaluation of the pdf response of dynamic systems subjected to non-stationary loads". Engineering Structures. 84(1), 2015, pp.419-429.

5. Eurocode C E N. 8. "Design provisions of structures for earthquake resistance, part 2: bridges. EN 1998-2-2005". European Committee for Standardization, Brussels, pp.253-255.

6. Chinese Standard. "Code for Seismic Design of Buildings, $G B$. 50011-2010". Beijing: Chinese Architectural Industry Press, China, pp.125-130.

7. Chinese M. C. Standard. "Guidelines for Seismic Design of Highway Bridges, JTG/T B02-01-2008". Beijing: China Communications Press, China, pp.133-138.

8. Lin, J. H.; Zhang, Y. H.; Li, Q. S.; Williams, F. W. "Seismic spatial effects for long-span bridges using the pseudo excitation method". Engineering Structures. 26(9), 2004, pp.1207-1216.

9. Kim, Gee-Cheol, and Joo-Won Kang. "Seismic Response Control of Dome Structure Subjected to Multi-Support Earthquake Excitation". Journal of the Korean Association for Spatial Structures. 14(4), 2014, pp.89-96.

10. Caprani, Colin C. "Application of the pseudo-excitation method to assessment of walking variability on footbridge vibration". Computers \& Structures. 132, 2014, pp.43-54.
11. Jia Hongyu, Zhang Deyi, Zheng Shixiong, Xie W C, Pandey M D. "Local site effects on a high-pier railway bridge under tridirectional spatial excitations: nonstationary stochastic analysis". Soil Dynamics and Earthquake Engineering. 52, 2013, pp.55-69.

12. Zanardo G, Hao H, Modena C. "Seismic response of multi-span simply supported bridges to a spatially varying earthquake ground motion". Earthquake Engineering and Structural Dynamics. 31(6), 2002, pp.1325-1345.

13. Su Cheng, $\mathrm{Xu}$ Rui. "Random vibration analysis of structures subjected to non-stationary excitations by time domain method". Engineering Mechanics. 27(12), 2010, pp.77-83. (in Chinese)

14. Xu Rui, Su Cheng. "Fast pseudo-excitation method in structural non-stationary stochastic response analysis". Chinese Journal of Computational Mechanics. 27(5), 2010, pp.822-827. (in Chinese)

15. Zhang Jin, Jia Hongyu, Zheng Shixiong et al. "Analysis of a highpier railway bridge under spatial stochastic stationary and nonstationary earthquake excitations". Tehnički vjesnik. 23(2), 2016, pp.465-475.

16. Zhang, De-Yi, et al. "A highly efficient and accurate stochastic seismic analysis approach for structures under tridirectional nonstationary multiple excitations". Computers \& Structures. 2014, 145 (1), pp.23-35.

17. Zhang, Y. H., et al. "Random vibration analysis of long-span structures subjected to spatially varying ground motions". Soil Dynamics and Earthquake Engineering. 29(4), 2009, pp.620-629.

18. Caughey T K, Stumpf H J. "Transient Response of a Dynamic System under Random Vibration". Journal of Applied Mechanics, 28(1), 1961, pp.563-566. 